\title{
Evaluation of Left Main Coronary Artery Using Optical Frequency Domain Imaging and Its Pitfalls
}

\author{
Vincent Roule $\mathbb{D}^{1},{ }^{1,2}$ Idir Rebouh, ${ }^{1}$ Adrien Lemaitre, ${ }^{1}$ Mathieu Bignon, ${ }^{1}$ Pierre Ardouin, ${ }^{1}$ \\ Rémi Sabatier, ${ }^{1}$ Fabien Labombarda, ${ }^{1,2}$ Katrien Blanchart, ${ }^{1}$ and Farzin Beygui ${ }^{1,2,3}$ \\ ${ }^{1} \mathrm{CHU}$ de Caen Normandie, Service de Cardiologie, Caen 14000, France \\ ${ }^{2}$ Normandie University, UNICAEN, EA 4650 Signalisation, \\ Électrophysiologie et imagerie des lésions d'ischémie-reperfusion myocardique, Caen 14000, France \\ ${ }^{3}$ ACTION Study Group, Cardiology Department, Pitié Salpêtrière University Hospital, Paris, France
}

Correspondence should be addressed to Vincent Roule; roule-v@chu-caen.fr

Received 29 February 2020; Accepted 21 May 2020; Published 12 June 2020

Academic Editor: Shenghua Zhou

Copyright ( 2020 Vincent Roule et al. This is an open access article distributed under the Creative Commons Attribution License, which permits unrestricted use, distribution, and reproduction in any medium, provided the original work is properly cited.

\begin{abstract}
Objectives. We aimed to assess the quality of optical frequency domain imaging (OFDI) of the left main (LM) arterial wall and describe and analyse potential artefacts in this setting. Background. OFDI is increasingly used to assess ambiguous lesions and optimize LM percutaneous coronary intervention. However, its ability to provide artefact-free high-quality images of coronary ostia and large segments such as the LM remains uncertain. Methods. We included 42 consecutive patients who underwent OFDI, including LM imaging. Each OFDI frame was subdivided into four quadrants and analysed. The number of quadrants with artifacts was calculated within the proximal, mid, and distal LM and the first $5 \mathrm{~mm}$ of the left anterior descending artery (LAD) and/or left circumflex artery (LCX). Results. The quadrants analysis showed an overall artifact rate of $8.9 \%$, mostly out-of-field (45.1\%) or residual blood (44.7\%) artefacts. Most artifacts were located in the proximal LM (18.6\%) with a stepwise reduction of artifact rates towards distal segments (mid LM 5.8\%; distal LM 3.6\%, ostial LAD 2.6\%, and ostial LCX 0\%; $p<0.001$ ). While 20 $(48.8 \%)$ patients had angiographically visible plaques, OFDI showed plaques in 32 patients $(76.2 \% ; p=0.007)$. Conclusion. OFDI can accurately evaluate the LM and detect and assess angiographically unvisualized atherosclerotic plaques providing accurate assessment of $>90 \%$ of the quadrants of the LM and the ostia of its bifurcation branches. However, artifacts mainly located in the proximal LM and decreasing distally in a stepwise fashion should be considered in the interpretation of OFDI in this setting.
\end{abstract}

\section{Introduction}

Accurate assessment of the left main (LM) coronary artery disease is crucial to determine treatment strategies and improve prognosis [1]. Significant LM stenosis accounts for $4.8 \%$ of all coronary angiograms and is rarely isolated [2]. The diagnosis of LM disease is based on coronary angiography in routine practice. However, due to a short vessel segment, lack of a reference segment in presence of diffuse atheroma, frequent overlapping daughter branches, and foreshortening [3-5], the accuracy of angiographic determination of LM narrowing may be limited [4]. Percutaneous coronary intervention (PCI) of unprotected LM has been increasingly performed over the last decade [6-8]. LM PCI is a high risk and challenging procedure which requires high precision given the large amount of myocardium at risk [1].

Optical coherence tomography (OCT) provides high image quality offering a unique insight into plaque characterisation and detailed structural information pre- and post-PCI [9]. Initially, OCT was not considered suitable for the assessment of the LM because of the large coronary size and poor blood washing $[10,11]$. The recently developed optical frequency domain imaging (OFDI) provides higher acquisition speed and larger field of view compared with prior generation time-domain OCT which may potentially overcome previous limitations. OCT and OFDI are increasingly used to assess ambiguous lesions and guide PCI [12]. Nevertheless, dedicated studies testing the possible 
pitfalls of OFDI imaging in LM remain scarce, especially in a population without previous LM stenting.

The aim of our study was to assess the quality of ODFI imaging and to describe and analyse its potential artefacts in the assessment of LM arterial wall in coronary artery disease patients with or without detectable angiographic LM lesions.

\section{Methods}

2.1. Study Population. We retrospectively included all consecutive patients who underwent OFDI including the whole LM and the ostia of its bifurcation branches in our center between May 2015 and August 2018. Patients with previous LM stenting were excluded.

\subsection{OFDI Image Analysis. The OFDI procedure} (Lunawave ${ }^{\circledR}$, FastView ${ }^{\circledR}$, Terumo Europe, Leuven, Belgium) was performed as previously described [13]. Images were analysed offline by 2 investigators (VR and IR) using previously validated criteria for OCT plaque characterisation [14-16]. We analysed the whole LM from the LM ostium or catheter tip to the ostia of its bifurcation branches defined as the first $5 \mathrm{~mm}$ of the left anterior descending artery (LAD) and/or left circumflex artery (LCX). LM length was obtained from OFDI longitudinal reconstructions and defined as the distance between the first distal frame of the LM at crosssectional image and the last proximal LM frame before aorta or catheter visualisation. Proximal, mid, and distal LM were defined, respectively, as the first, the second, and the last third of the whole LM [17]. Reference lumen area and percent area stenosis were calculated as previously described [18]. When atherosclerotic plaque was identified, at least 3 measurements of the intima and media thickness were performed where the plaque was largest. When disappearance of the media was observed, a value of $10 \mu \mathrm{m}$ (normal value usually observed in our practice) was given to allow calculation of the intima/media ratio.

The feasibility of OFDI assessment was measured in the following regions: proximal LM, mid LM, distal LM, and the ostia of its bifurcation branches [17]. For each region, we analysed all frames. Each frame was subdivided into four quadrants: quadrant $1=0-90^{\circ}$, quadrant $2=90-180^{\circ}$, quadrant $3=180-270^{\circ}$, and quadrant $4=270-360^{\circ}$. The number of fully assessable quadrants per frame and consequently quadrants with artifacts were calculated [19]. Artifacts were defined as follows:

(i) Quadrants out of the field of view: "out-of-screen" loss of image;

(ii) Residual blood: suboptimal vessel flushing causing signal-rich blood swirls in the lumen. Residual blood attenuates the OCT light beam and may defocus the beam if red cell density is high;

(iii) Sew-up or seam artifacts: rapid movement of the artery or the imaging catheter during the acquisition of a single cross-sectional image causing misalignment along the circumference of the image; (iv) Related to eccentric wire position: when the vessel is large in size and in vessel curvature, the catheter alignment could be noncoaxial, and the resulting image appears elliptical. This makes the measurements inaccurate.

2.3. Quantitative Coronary Angiography. Quantitative coronary angiography (QCA) was performed offline (CAAS II, Pie Medical, Maastricht, Netherlands) using validated quantitative methods [20]. The following angiographic data were calculated: vessels reference diameter, percentage diameter stenosis, and LM length.

2.4. Statistical Analysis. Continuous and categorical variables were expressed as mean \pm standard deviation and numbers of patients and percentages and compared between groups identified by the presence or not of at least one artefact using Student's test or the chi square test, respectively. A $p$ value of $<0.05$ was considered statistically significant. SAS software version 9.4 (SAS Institute, Cary, NC) was used for statistical analysis.

\section{Results}

A total of 42 patients fulfilled the inclusion criteria. LM OFDI was performed to evaluate ambiguous LM lesions in 14 and as a part of a long run for other patients. Baseline clinical and angiographic characteristics are reported in Table 1 . Most patients presented with myocardial infarction $(n=38 ; 90.5 \%)$ and had single vessel disease $(n=26 ; 61.9 \%)$.

OFDI analysis is reported in Table 2. Atherosclerotic disease preferentially affected distal LM $(n=13,40.6 \%)$. The quadrants analysis showed an overall artifact rate of $8.9 \%$, which was significantly different across the considered LM segments ( $p<0.001$, Figure 1$)$. Most artifacts were located in the proximal LM (18.6\%) and rates decreased distally in a stepwise fashion (mid LM 5.8\%; distal LM 3.6\%, ostial LAD 2.6\%, and ostial LCX 0\%). There was a trend towards shorter LM length assessed with OFDI as compared with QCA $(10.1 \pm 4.5$ vs $12 \pm 4.38 \mathrm{~mm}$, respectively; $p=0.0504$ ). Most artifacts (Figure 2) were related to quadrants out of the field of view and residual blood $(45.1 \%$ and $44.7 \%$, respectively). Other artefacts were sew-up or seam artifacts $(7.2 \%)$ or related to eccentric wire position (3\%). The only variable significantly associated with higher rates of artefacts was the length of LM (11.3 \pm 4.8 vs $7.9 \pm 2.8 \mathrm{~mm} ; \quad p=0.016$; Supplementary Table S1). The correlation between artifact's rate and LM diameter was low $(r=0.34 ; p=0.026)$.

While 20 patients (48.8\%) had angiographic signs of LM atherosclerosis, OFDI analysis showed atherosclerosis in 32 patients $(76.2 \% ; p=0.007)$. As shown in Table 3, plaques were fibrous $(n=22 ; 68.7 \%)$ or fibrocalcific $(n=10 ; 31.3 \%)$ and presented intimal hyperplasia $(0.53 \pm 0.22$ vs $0.10 \pm 0.02 \mathrm{~mm}, p<0.001)$ when compared to normal segments (Figure 3). 
TABLE 1: Baseline clinical and angiographic characteristics of the study population.

\begin{tabular}{lc}
\hline & All $(n=42)$ \\
\hline Baseline characteristics & \\
Age (years) & $55.6 \pm 16$ \\
Men & $26(61.9 \%)$ \\
Body mass index $\left(\mathrm{kg} / \mathrm{m}^{2}\right)$ & $25.9 \pm 4.7$ \\
Systemic hypertension & $15(35.2 \%)$ \\
Hyperlipidemia & $15(35.2 \%)$ \\
Active smoker & $25(59.5 \%)$ \\
Diabetes mellitus & $4(9.5 \%)$ \\
History of & \\
$\quad$ Myocardial infarction & $4(9.5 \%)$ \\
CABG & $0(0 \%)$ \\
PCI & $5(11.9 \%)$ \\
Baseline eGFR <60 ml/min & $3(7.1 \%)$ \\
Left ventricular ejection fraction (\%) & $53.1 \pm 10.5$ \\
\hline Clinical presentation & $4(9.5 \%)$ \\
Stable angina & $10(23.8 \%)$ \\
Non-ST-elevation myocardial infarction & $28(66.7 \%)$ \\
ST-elevation myocardial infarction & \\
\hline Angiographic characteristics & \\
Guiding catheter & $38(90.5 \%)$ \\
Extra backup & $4(9.5 \%)$ \\
Judkins left guiding catheter & $26(61.9 \%)$ \\
Single-vessel disease & $9(21.4 \%)$ \\
Two-vessel disease & $7(16.7 \%)$ \\
Three-vessel disease & $12 \pm 4.38$ \\
LM length (mm) & $4.1 \pm 0.56$ \\
LM reference diameter (mm) & $3.1 \pm 0.34$ \\
Proximal LAD reference diameter (mm) & $2.8 \pm 0.38$ \\
Proximal LCX reference diameter (mm) & $25 \pm 16[5-70]$ \\
Angiographic signs of LM atherosclerosis & glomerular fil- \\
LM stenosis (\%) [range] & left circumflex \\
\hline CABG = coronary artery bypass graft; eGFR = estimated & \\
tration rate; LAD = left anterior descending artery; LCX & \\
artery; LM = left main; PCI = percutaneous coronary intervention. \\
\end{tabular}

\section{Discussion}

Our study showed that overall more than $90 \%$ of the quadrants of LM were adequately assessable using OFDI. OFDI detected almost $60 \%$ more atherosclerotic plaques than angiography. Artefact rates were low and significantly different between analysed segments, most artifacts being located in the proximal LM. Artefacts were mainly due to quadrants out of the field of view or residual blood effect. When present, plaques were preponderantly fibrous or fibrocalcific with intimal hyperplasia on OFDI analysis.

The angiographic evaluation of LM disease severity can be doubtful or discordant between angiographic views or between operators because of the inherent limitations of angiographic assessment at this site [3-5]. OCT and OFDI are increasingly used to assess doubtful lesions and optimize complex PCI [12]. However, their ability to provide highquality images in ostial and large calibre segments such as left main coronary artery remains challenged. Our study showed that overall more than $90 \%$ of the quadrants of the LM were adequately assessable by OFDI. Previous studies in the setting of pre- or post-PCI have reported poorer performances of
TABLE 2: OFDI analysis of the study population.

\begin{tabular}{|c|c|}
\hline & All $(n=42)$ \\
\hline \multicolumn{2}{|l|}{ OFDI characteristics } \\
\hline LM length (mm) & $10.1 \pm 4.5$ \\
\hline LM reference LA $\left(\mathrm{mm}^{2}\right)$ & $14.9 \pm 4.8$ \\
\hline Proximal LAD reference LA $\left(\mathrm{mm}^{2}\right)$ & $7.8 \pm 3.2$ \\
\hline Proximal LCX reference LA $\left(\mathrm{mm}^{2}\right)$ & $7.8 \pm 4.6$ \\
\hline OFDI signs of LM atherosclerosis & $32(76.2 \%)$ \\
\hline LM stenosis (\%) [range] & $26 \pm 18[7-76]$ \\
\hline \multicolumn{2}{|l|}{ Tightest lesion site } \\
\hline Proximal LM & $3(9.4 \%)$ \\
\hline Mid LM & $1(3.1 \%)$ \\
\hline Distal LM & $13(40.6 \%)$ \\
\hline Ostial LAD & $11(34.4 \%)$ \\
\hline Ostial LCX & $4(12.5 \%)$ \\
\hline \multicolumn{2}{|l|}{ OFDI imaging analysis } \\
\hline \multicolumn{2}{|l|}{ Global LM analysis } \\
\hline Quadrants with artifacts/total quadrants & $1207 / 13540$ \\
\hline Artifacts (\%) [range] & $8.9[0-55.6]$ \\
\hline Analyzable quadrants per frame & $3.6 \pm 0.5$ \\
\hline \multicolumn{2}{|l|}{ Proximal LM } \\
\hline Quadrants with artifacts/total quadrants & $835 / 4472$ \\
\hline Artifacts (\%) [range] & $18.6[0-100]$ \\
\hline Analyzable quadrants per frame & $3.3 \pm 1$ \\
\hline \multicolumn{2}{|l|}{ Mid LM } \\
\hline Quadrants with artifacts/total quadrants & $223 / 4516$ \\
\hline Artifacts (\%) [range] & $5.8[0-62.5]$ \\
\hline Analyzable quadrants per frame & $3.8 \pm 0.5$ \\
\hline \multicolumn{2}{|l|}{ Distal LM } \\
\hline Quadrants with artifacts/total quadrants & $149 / 4552$ \\
\hline Artifacts (\%) [range] & $3.6[0-46.6]$ \\
\hline Analyzable quadrants per frame & $3.9 \pm 0.4$ \\
\hline \multicolumn{2}{|l|}{ Ostial LAD } \\
\hline Quadrants with artifacts/total quadrants & $172 / 5800$ \\
\hline Artifacts (\%) [range] & $2.6[0-47.8]$ \\
\hline Analyzable quadrants per frame & $3.9 \pm 0.4$ \\
\hline \multicolumn{2}{|l|}{ Ostial LCX } \\
\hline Quadrants with artifacts/total quadrants & $0 / 1124$ \\
\hline Artifacts (\%) [range] & $0[0-0]$ \\
\hline Analyzable quadrants per frame & $0 \pm 0$ \\
\hline
\end{tabular}

$\mathrm{CABG}=$ coronary artery bypass graft; eGFR $=$ estimated glomerular filtration rate; $\mathrm{LA}=$ lumen area; $\mathrm{LAD}=$ left anterior descending artery; LCX = left circumflex artery; $L M=$ left main; OFDI = optical frequency domain imaging

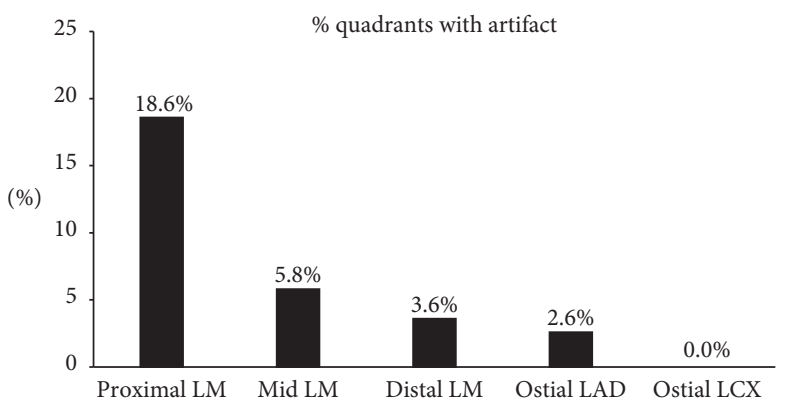

Figure 1: Percentage of quadrants with artifacts in the different explored segments, $p<0.0001$. LAD, left anterior descending; LCX, left circumflex; LM, left main. 


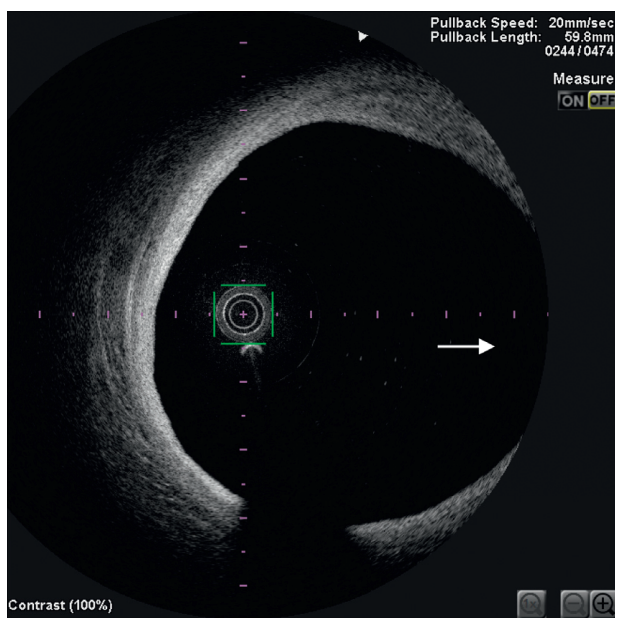

(a)

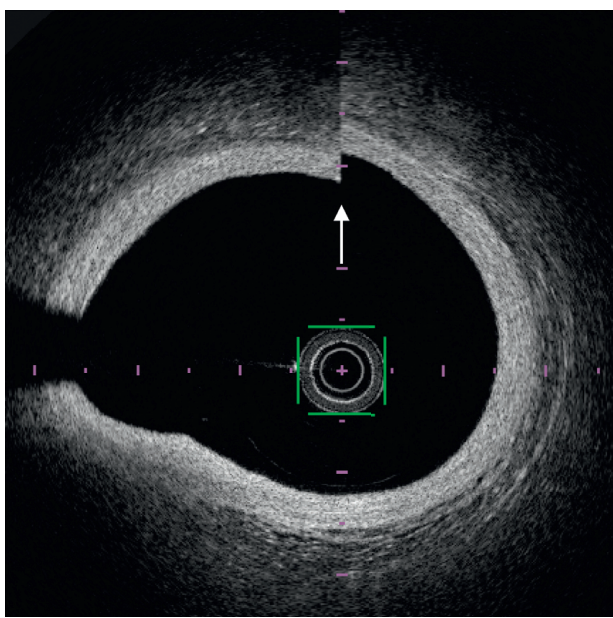

(c)

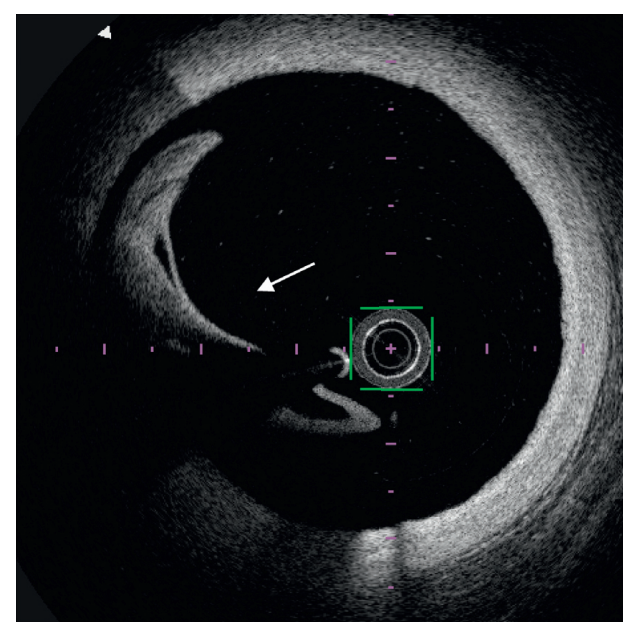

(b)

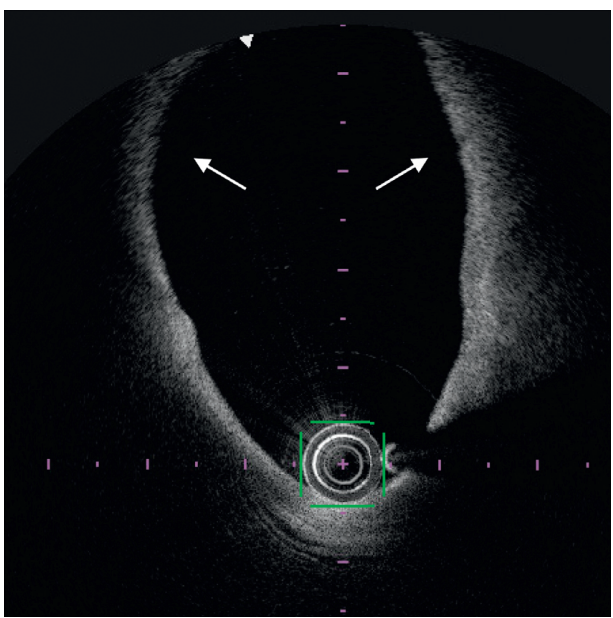

(d)

Figure 2: Optical frequency domain imaging examples of artifacts: quadrants out of the field of view (white arrow) (a), incomplete blood displacement by iodine contrast producing volute (white arrow) with high attenuation of optical signal (b), sew-up artifact (c) (white arrow), and artifact related to eccentric wire position causing elliptical image and signal attenuation (d) (white arrows).

TABLE 3: OFDI analysis of the underlying plaque.

\begin{tabular}{|c|c|c|}
\hline OFDI characteristics of the plaque & \multicolumn{2}{|c|}{ Diseased LM $(n=32)$} \\
\hline Fibrous plaque & & \\
\hline Fibrocalcific plaque & & \\
\hline Cholesterol crystals & & \\
\hline Thrombus & & \\
\hline Microchannels & & \\
\hline Disappearance of media & & \\
\hline OFDI quantitative analysis & Normal LM $(n=10)$ & Diseased LM $(n=32)$ \\
\hline Intima thickness $(\mathrm{mm})^{*}$ & $0.10 \pm 0.02$ & $0.53 \pm 0.22$ \\
\hline Media thickness (mm) & $0.10 \pm 0.02$ & $0.14 \pm 0.10$ \\
\hline Intima/media ratio* & $1.0 \pm 0.17$ & $4.70 \pm 2.51$ \\
\hline
\end{tabular}

$\mathrm{CABG}=$ coronary artery bypass graft; eGFR = estimated glomerular filtration rate; $\mathrm{LA}=$ lumen area; $\mathrm{LAD}=$ left anterior descending artery; LCX $=$ left circumflex artery; $\mathrm{LM}=$ left main; OFDI $=$ optical frequency domain imaging. ${ }^{*} p<0.001$ diseased vs normal LM.

OCT in assessing the LM $[19,21]$. The most important pitfall reported in one study before PCI was the ability of FD-OCT to fully assess ostial LM in only $12.5 \%$ of cases [21]. Parodi et al., who studied LM stenting in 15 patients, reported that $69 \pm 20 \%$ of the stent inner area (or $2.7 \pm 0.8$ quadrants/slice) were analyzable, mostly because of quadrants out of screen [19]. The small cohorts and the different OCT systems may participate to the different reported performances. We used OFDI technology which provides a better signal-to-noise ratio, higher acquisition speed during automatic motorized 


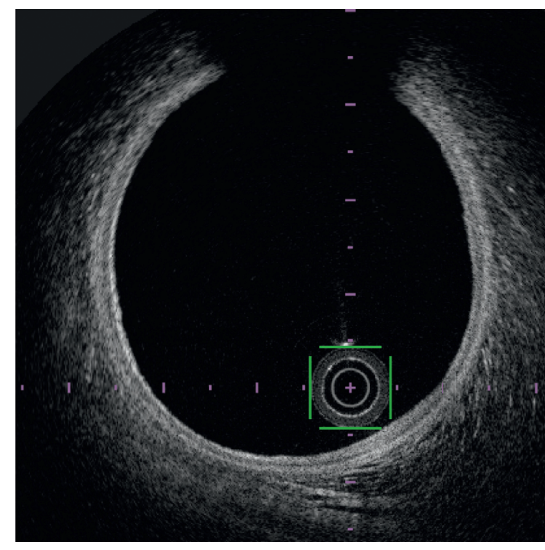

(a)

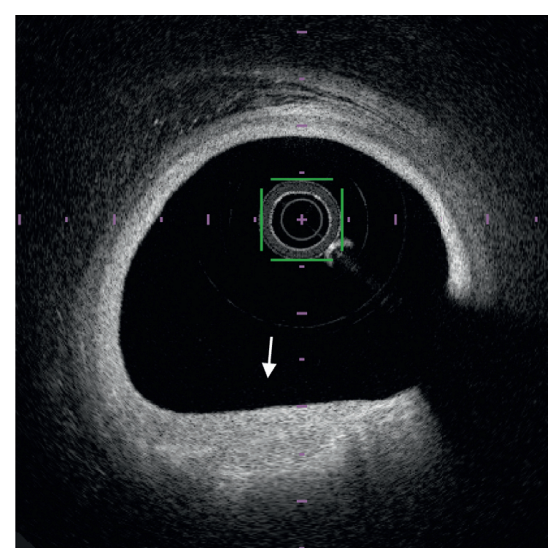

(b)

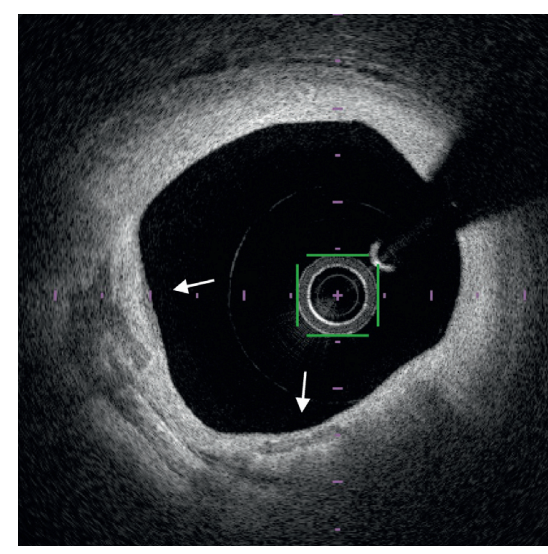

(c)

FIGURE 3: Optical frequency domain imaging examples of plaque analysis: normal LM (a), fibrous plaque (b) (white arrow), and fibrocalcific plaque (c) (white arrows) with intimal hyperplasia and disappearance of the media.

pullback, and larger field of view as compared to latter OCT systems [22]. Additionally, our patients did not have tight stenoses as the OFDI catheter may not cross tight calcified lesions in rare cases.

Most artifacts were located at the proximal part of the LM, as previously reported [21]. This is mostly due to incomplete blood displacement by iodine contrast which is more difficult to obtain for large and proximal segments and quadrants out of the imaging field of view. Other observed image artifacts were sew-up artifacts as the result of rapid artery or imaging system movements during the acquisition of a single cross-sectional image and eccentricity artefact due to the wire position causing elliptical images that attenuate the signal [22]. Nonuniform rotational distortion, fold-over, air bubbles, and saturation artifacts were not observed in our study but may still occur with OFDI.

OFDI provided a more precise evaluation of LM atherosclerotic plaques undetected by angiography in one-third of our patients. OFDI showed mostly fibrous or fibrocalcific plaques with intimal hyperplasia in concordance with previous intravascular ultrasound studies [23, 24]. The lipid core content of LM lesions is less important than in other coronary segments $[23,24]$ and may explain the greater ability of OFDI to assess the LM wall in absence of attenuation of optical signal beyond the lipid-rich plaques. We found that atherosclerotic disease preferentially affected distal LM encroaching LAD or LCX ostia. Indeed, LM disease is rather diffuse than focal $[25,26]$ and this point should be considered when treating LM stenosis, as distal disease is associated with worse outcome [27, 28].

Our study showed that OFDI can adequately evaluate LM bifurcation which is the most commonly diseased segment of the LM [26]. On the contrary, operators should consider the limitations of OFDI to assess the ostial LM wall. Tortuous, very large, and short LM may not be ideal for OFDI evaluation. For ostial LM assessment, the choice of the guiding catheter may differ [3]. We preferentially used an extra backup (EBU) guiding catheter which allowed deeper engagement of the LM and consequently adequate contrast flushing. But the true anatomical ostium may have been missed as supported by the shorter LM length observed with OFDI compared to angiography. This is important for optimal stent sizing as malapposition is more common in the proximal than distal LM [29]. The Judkins left (3.5 or 4) guiding catheter can be more easily positioned at the ostium but at the cost of lower flushing and image quality as well as less support in case of further PCI.

4.1. Limits. Although larger than most studies on the subject, our study is based on a relatively small sized cohort. We did not assess patients with ostial or very severe LM stenosis which are usually not suitable for OFDI assessment.

\section{Conclusion}

Our study showed that OFDI can accurately evaluate the LM and detect and assess angiographically unvisualized atherosclerotic plaques, providing accurate assessment of $>90 \%$ of the quadrants of the LM and the ostia of its bifurcation branches. Most artifacts were located in the proximal LM and their rate decreased distally. OFDI can accurately evaluate LM bifurcation, which is the most commonly diseased segment for LM stenosis, and provides a precise evaluation of LM atherosclerotic plaques. However, out-of-field and residual blood-related artifacts should be considered when using OFDI in the ostial or proximal LM.

\section{Data Availability}

The datasets used and/or analysed during the current study are available from the corresponding author upon reasonable request.

\section{Conflicts of Interest}

The authors have no conflicts of interest to declare.

\section{Supplementary Materials}

Supplementary Table S1: comparison between patients with and without artefacts on OFDI analysis. (Supplementary Materials) 


\section{References}

[1] A. A. El-Menyar, J. Al Suwaidi, and D. R. Holmes Jr., "Left main coronary artery stenosis: state-of-the-art," Current Problems in Cardiology, vol. 32, no. 3, pp. 103-193, 2007.

[2] G. D. Giannoglou, A. P. Antoniadis, Y. S. Chatzizisis, E. Damvopoulou, G. E. Parcharidis, and G. E. Louridas, "Prevalence of narrowing > or $=50 \%$ of the left main coronary artery among 17,300 patients having coronary angiography," The American Journal of Cardiology, vol. 98, no. 9, pp. 1202-1205, 2006.

[3] R. Bing, A. S. C. Yong, and H. C. Lowe, "Percutaneous transcatheter assessment of the left main coronary artery," JACC: Cardiovascular Interventions, vol. 8, no. 12, pp. 15291539, 2015.

[4] J. M. Isner, J. Kishel, K. M. Kent, J. A. Ronan Jr., A. M. Ross, and W. C. Roberts, "Accuracy of angiographic determination of left main coronary arterial narrowing. Angiographic-histologic correlative analysis in 28 patients," Circulation, vol. 63, no. 5, pp. 1056-1064, 1981.

[5] C. W. White, C. B. Wright, D. B. Doty et al., "Does visual interpretation of the coronary arteriogram predict the physiologic importance of a coronary stenosis?" New England Journal of Medicine, vol. 310, no. 13, pp. 819-824, 1984.

[6] J. Mehilli, A. Kastrati, R. A. Byrne et al., "Paclitaxel- versus sirolimus-eluting stents for unprotected left main coronary artery disease," Journal of the American College of Cardiology, vol. 53, no. 19, pp. 1760-1768, 2009.

[7] T. Palmerini, P. Serruys, A. P. Kappetein et al., "Clinical outcomes with percutaneous coronary revascularization vs coronary artery bypass grafting surgery in patients with unprotected left main coronary artery disease: a meta-analysis of 6 randomized trials and 4,686 patients," American Heart Journal, vol. 190, pp. 54-63, 2017.

[8] P. S. Teirstein and M. J. Price, "Left main percutaneous coronary intervention," Journal of the American College of Cardiology, vol. 60, no. 17, pp. 1605-1613, 2012.

[9] F. Prati, G. Guagliumi, G. S. Mintz et al., "Expert review document part 2: methodology, terminology and clinical applications of optical coherence tomography for the assessment of interventional procedures," European Heart Journal, vol. 33, no. 20, pp. 2513-2520, 2012.

[10] R. Hamdan, R. G. Gonzalez, S. Ghostine, and C. Caussin, "Optical coherence tomography: from physical principles to clinical applications," Archives of Cardiovascular Diseases, vol. 105, no. 10, pp. 529-534, 2012.

[11] F. Prati, E. Regar, G. S. Mintz et al., "Expert review document on methodology, terminology, and clinical applications of optical coherence tomography: physical principles, methodology of image acquisition, and clinical application for assessment of coronary arteries and atherosclerosis," European Heart Journal, vol. 31, no. 4, pp. 401-415, 2010.

[12] K. C. Koskinas, G. J. Ughi, S. Windecker, G. J. Tearney, and L. Räber, "Intracoronary imaging of coronary atherosclerosis: validation for diagnosis, prognosis and treatment," European Heart Journal, vol. 37, no. 6, pp. 524-535, 2016.

[13] H. M. Garcia-Garcia, T. Muramatsu, S. Nakatani et al., "Serial optical frequency domain imaging in STEMI patients: the follow-up report of TROFI study," European Heart Journal-Cardiovascular Imaging, vol. 15, no. 9, pp. 987-995, 2014.

[14] I.-K. Jang, G. J. Tearney, B. MacNeill et al., "In vivo characterization of coronary atherosclerotic plaque by use of optical coherence tomography," Circulation, vol. 111, no. 12, pp. 1551-1555, 2005.

[15] H. Jia, F. Abtahian, A. D. Aguirre et al., "In vivo diagnosis of plaque erosion and calcified nodule in patients with acute coronary syndrome by intravascular optical coherence tomography," Journal of the American College of Cardiology, vol. 62, no. 19, pp. 1748-1758, 2013.

[16] G. J. Tearney, E. Regar, T. Akasaka et al., "Consensus standards for acquisition, measurement, and reporting of intravascular optical coherence tomography studies," Journal of the American College of Cardiology, vol. 59, no. 12, pp. 1058-1072, 2012.

[17] F. Burzotta, I. Dato, C. Trani et al., "Frequency domain optical coherence tomography to assess non-ostial left main coronary artery," EuroIntervention, vol. 10, no. 9, pp. e1-e8, 2015.

[18] N. Amabile, S. Hammas, S. Fradi et al., "Intra-coronary thrombus evolution during acute coronary syndrome: regression assessment by serial optical coherence tomography analyses," European Heart Journal-Cardiovascular Imaging, vol. 16, no. 4, pp. 433-440, 2015.

[19] G. Parodi, A. Maehara, G. Giuliani et al., "Optical coherence tomography in unprotected left main coronary artery stenting," EuroIntervention, vol. 6, no. 1, pp. 94-99, 2010.

[20] P. Garrone, G. Biondi-Zoccai, I. Salvetti et al., "Quantitative coronary angiography in the current era: principles and applications," Journal of Interventional Cardiology, vol. 22, no. 6, pp. 527-536, 2009.

[21] Y. Fujino, H. G. Bezerra, G. F. Attizzani et al., "Frequencydomain optical coherence tomography assessment of unprotected left main coronary artery disease-a comparison with intravascular ultrasound," Catheterization and Cardiovascular Interventions, vol. 82, no. 3, pp. E173-E183, 2013.

[22] H. G. Bezerra, M. A. Costa, G. Guagliumi, A. M. Rollins, and D. I. Simon, "Intracoronary optical coherence tomography: a comprehensive review," JACC: Cardiovascular Interventions, vol. 2, no. 11, pp. 1035-1046, 2009.

[23] N. Mercado, T. Moe, M. Pieper et al., "Tissue characterisation of atherosclerotic plaque in the left main: an in vivo intravascular ultrasound radiofrequency data analysis," EuroIntervention, vol. 7, no. 3, pp. 347-352, 2011.

[24] J. J. Wykrzykowska, G. S. Mintz, H. M. Garcia-Garcia et al., "Longitudinal distribution of plaque burden and necrotic core-rich plaques in nonculprit lesions of patients presenting with acute coronary syndromes," JACC: Cardiovascular Imaging, vol. 5, no. 3, pp. S10-S18, 2012.

[25] C. Oviedo, A. Maehara, G. S. Mintz et al., "Intravascular ultrasound classification of plaque distribution in left main coronary artery bifurcations," Circulation: Cardiovascular Interventions, vol. 3, no. 2, pp. 105-112, 2010.

[26] M. Ragosta, S. Dee, I. J. Sarembock, L. C. Lipson, L. W. Gimple, and E. R. Powers, "Prevalence of unfavorable angiographic characteristics for percutaneous intervention in patients with unprotected left main coronary artery disease," Catheterization and Cardiovascular Interventions, vol. 68, no. 3, pp. 357-362, 2006.

[27] T. Naganuma, A. Chieffo, E. Meliga et al., "Long-term clinical outcomes after percutaneous coronary intervention for ostial/ mid-shaft lesions versus distal bifurcation lesions in unprotected left main coronary artery," JACC: Cardiovascular Interventions, vol. 6, no. 12, pp. 1242-1249, 2013.

[28] M. Valgimigli, P. Malagutti, G. A. Rodriguez-Granillo et al., "Distal left main coronary disease is a major predictor of outcome in patients undergoing percutaneous intervention in 
the drug-eluting stent era," Journal of the American College of Cardiology, vol. 47, no. 8, pp. 1530-1537, 2006.

[29] Y. Fujino, G. F. Attizzani, H. G. Bezerra et al., "Serial assessment of vessel interactions after drug-eluting stent implantation in unprotected distal left main coronary artery disease using frequency-domain optical coherence tomography," JACC: Cardiovascular Interventions, vol. 6, no. 10, pp. 1035-1045, 2013. 